\title{
Future-looking Logics on Data Words and Trees
}

\author{
Diego Figueira and Luc Segoufin \\ INRIA, LSV, ENS Cachan, France
}

\begin{abstract}
In a data word or a data tree each position carries a label from a finite alphabet and a data value from an infinite domain. Over data words we consider the logic $L_{T L}^{\downarrow}(F)$, that extends $\operatorname{LTL}(F)$ with one register for storing data values for later comparisons. We show that satisfiability over data words of $\operatorname{LTL}_{1}^{\downarrow}(\mathrm{F})$ is already non primitive recursive. We also show that the extension of $L T L_{1}^{\downarrow}(F)$ with either the backward modality $\mathrm{F}^{-1}$ or with one extra register is undecidable. All these lower bounds were already known for $\operatorname{LTL}_{1}^{\downarrow}(\mathrm{X}, \mathrm{F})$ and our results essentially show that the $\mathrm{X}$ modality was not necessary.

Moreover we show that over data trees similar lower bounds hold for certain fragments of XPath.
\end{abstract}

\section{Introduction}

A data word (data tree) is a word (tree) where each position carries a label from a finite alphabet and a datum from some infinite domain. These models have been considsered in the realm of semistructured data [3], timed automata [5] and extended temporal logics $98 / 12$. In this work we consider an infinite domain with no structure where we can only test for equality or inequality between elements.

There have been various logics considered to specify properties over data words and data trees. For example from the standpoint of Temporal Logics (both on data words [8] and trees [12]), of First Order Logics (see [4] for the data words case and [3] for trees), or of logics based on tree patterns [7]2]. The logic $L_{L}^{\downarrow}(X, F)$ is the extension of $\operatorname{LTL}(X, F)$ with the ability to use one register for storing a data value for later comparisons. It has been studied in 98 where satisfiability and expressivity issues have been addressed. In [8] it has been established that the satisfiability problem for $L T L \downarrow 1$ non primitive recursive on data words. It is also shown in 8 that the two way extension $\mathrm{LTL}_{1}^{\downarrow}\left(\mathrm{X}, \mathrm{F}, \mathrm{F}^{-1}\right)$ is undecidable over data words and, similarly, that the extension to 2 registers $\mathrm{LTL}_{2}^{\downarrow}(\mathrm{X}, \mathrm{F})$ is undecidable.

Here we show that even without the $\mathbf{X}$ modality, all the aforementioned lower bounds remain valid: the satisfiability problem for $L T L_{1}^{\downarrow}(F)$ over data words is non primitive recursive, while for $L T L_{1}^{\downarrow}\left(F, F^{-1}\right)$ and $L T L_{2}^{\downarrow}(F)$ is undecidable.

Data trees can be seen as a coding of an XML document [312]. Therefore XPath, the node selecting language of W3C [6], can be considered as a logic over

\footnotetext{
* The authors acknowledge the financial support of the Future and Emerging Technologies (FET) programme within the Seventh Framework Programme for Research of the European Commission, under the FET-Open grant agreement FOX, number FP7-ICT-233599.
} 
data trees. By XPath, we refer to the 1.0 specification without all the domain specific features (arithmetic, string manipulation, etc.) As XPath is at the core of many XML standard languages (like XQuery and XSLT), deciding satisfiability of some of its fragments can be of great help during optimization stages.

A data word can be seen as a special case of an unranked ordered data tree, for instance by adding a root that is the parent of all the positions of the data word. With this consideration the fragment XPath $\left(\rightarrow, \rightarrow^{+},=\right)$of XPath that contains only the axis next-sibling $(\rightarrow)$ and following-sibling $\left(\rightarrow^{+}\right)$can be seen as a fragment of $L_{T L}^{\downarrow}(X, F)$. There are nonetheless two important differences between the expressive power of $\operatorname{XPath}\left(\rightarrow, \rightarrow^{+},=\right)$and $\operatorname{LTL}_{1}^{\downarrow}(\mathrm{X}, \mathrm{F})$. The first one is that the axis $\rightarrow^{+}$corresponds to the strict future modality in LTL, denoted $F_{s}$ in the sequel. Of course $\mathrm{F}$ can be defined using $F_{s}$ but the opposite is not true in the absence of $X$. The second difference lies in the fact that XPath can compare data values in a way strictly more limited than $L_{T L} L_{1}^{\downarrow}$. We illustrate this with an example. At a position of a data word, $\mathrm{LTL}_{1}^{\downarrow}(\mathrm{F})$ can store the current data value in the register, check for a later symbol a with a data value different from the one in the register, and then further check for a symbol $\mathrm{b}$ with a data value matching the one of the register. In this spirit, XPath could only compare the data values at the beginning and the end of the path and could not say anything about the data value of the intermediate a symbol. Hence XPath $\left(\rightarrow^{+},=\right)$should be seen as a fragment of $L T L_{1}^{\downarrow}\left(F_{s}\right)$, incomparable with $L T L_{1}^{\downarrow}(F)$ in terms of expressive power.

Based on these ideas, we exhibit a syntactic fragment $s L T L_{1}^{\downarrow}\left(F_{s}\right)$ of $L T L_{1}^{\downarrow}\left(F_{s}\right)$ that limits the use of register comparisons and has the same expressive power as $\operatorname{XPath}\left(\rightarrow^{+},=\right)$on data words. We then show that satisfiability over data words of $s L T L L_{1}^{\downarrow}\left(F_{s}\right)$ is non primitive recursive. Similarly the same restriction on $\mathrm{LTL}_{1}^{\downarrow}\left(\mathrm{F}_{\mathrm{s}}, \mathrm{F}_{\mathrm{s}}{ }^{-1}\right)$ yields a logic with the same expressive power as XPath $\left(\rightarrow^{+},{ }^{+} \leftarrow,=\right)$, which is shown to be undecidable.

Our non primitive recursive results are proved by a reduction from the emptiness problem of faulty counter automata. These are counter automata that may have incrementing errors in their counters during the run. Non-emptiness for this class of automata was proven to be decidable and not primitive recursive [16. Our reduction will be centered in a strategy of using data values for coding with some limitations- a next step move of this automaton. We show that the strict future modality together with the limited data comparison capabilities of $s L T L_{1}^{\downarrow}\left(F_{s}\right)$ are sufficient for our coding. With the extra power of $L T L_{1}^{\downarrow}(F)$ for comparing data values, we also show that strictness of the future modality can be avoided. Similar ideas are used for our undecidability results: The extra available expressive power is sufficient to forbid the incrementing errors and thus to code the emptiness problem of a Minsky Counter Automaton.

Related work. There are known complexity results concerning the satisfiability problem of several data-aware fragments of XPath on data trees. When all navigational axes are present, XPath is undecidable [11. When all vertical axes are present but in the absence of any horizontal axis, the status of the decidability of XPath $\left(\downarrow, \downarrow^{+}, \uparrow, \uparrow^{+},=\right)$is not yet known [1]. In this paper we show that if decid- 
able, the complexity of this fragment cannot be primitive recursive, even in the absence of the axes $\downarrow$ and $\uparrow$.

In [3] decidability over data trees of the two-variable fragment of first order logic, $\mathrm{FO}_{2}(+1, \sim)$ is established. As a direct consequence, a fragment of XPath without any descendant/ancestor or following/previous-sibling axes in the paths of the data test expressions, is shown to be decidable. On the opposite, in the present work we focus on the satisfiability under the absence of the successor axis.

In 12 it is shown that the emptiness problem for alternating automata with one register over data trees is decidable. As a direct consequence it is shown that XPath $\left(\downarrow, \downarrow^{+}, \rightarrow, \rightarrow^{+},=\right)$, with some restriction on the expressions with a data value test, is decidable and non primitive recursive. As a consequence of the present work, the hardness result already holds for XPath $\left(\downarrow^{+}, \rightarrow,=\right)$ and for $\mathrm{XPath}\left(\rightarrow^{+},=\right)$.

We finally remark that, nevertheless, the satisfiability of $\operatorname{XPath}\left(\downarrow^{+}, \downarrow^{+},=\right)$is "only" ExpTime-complete [10].

\section{Preliminaries}

We fix a finite set $\Sigma$ of labels and an infinite set $D$ of data values. The models we consider are either data words or data trees. A data word $\sigma$ over a finite alphabet $\Sigma$ is a non-empty word of $(\Sigma \times D)^{*}$. We assume no structure on $D$ and $D$ will be used only to perform tests for (in)equalities. In our examples we will always use data values from $\mathbb{N}$ seen as a set of numbers. The data trees we will be using are unranked and ordered. The domain of an unranked ordered tree is represented by a prefix-closed set $T$ of elements from $\mathbb{N}^{*}$ such that whenever $n(i+1) \in T$ then $n i \in T$. The elements of $T$ will be called nodes of the tree. A data tree $t$ over $\Sigma, D$ is a tree domain $T$ together with a labeling function $\lambda$ assigning an element of $\Sigma \times D$ to any node of $t$. We use the standard terminology for trees such as descendant, ancestor, sibling, etc.

LTL with registers. The most expressive logic for data words we treat here is $\mathrm{LTL}_{n}^{\downarrow}\left(\mathrm{F}, \mathrm{X}, \mathrm{F}^{-1}, \mathrm{X}^{-1}\right)$, the Linear Temporal Logic with the freeze quantifier $\left(\downarrow_{i}\right)$, test predicate $\left(\uparrow_{i}\right)$ and next $(\mathrm{X})$ and future $(\mathrm{F})$ temporal operators together with their inverse modalities $\left(\mathrm{X}^{-1}, \mathrm{~F}^{-1}\right)$. Sentences are defined:

$$
\varphi, \psi::=a\left|\downarrow_{i} \varphi\right| \uparrow_{i}|\neg \varphi| \varphi \wedge \psi|\varphi \vee \psi| \mathcal{O} \varphi
$$

where $a$ is a symbol from a finite alphabet $\Sigma, i \in\{1, \ldots, n\}$, and $\mathcal{O}$ ranges over $\left\{\mathrm{F}, \mathrm{X}, \mathrm{F}^{-1}, \mathrm{X}^{-1}\right\}$. We also use the strict future temporal operator $\mathrm{F}_{\mathrm{s}}$ as a shortcut for XF. We will consider fragments of this logics as simple restrictions to certain temporal operators. Intuitively, in the evaluation of $\downarrow_{i} \varphi$, current data value is 'saved' in the register $i$, and any appearance of $\uparrow_{i}$ in $\varphi$ holds at a position iff its datum is equal to the one stored in register $i$.

Given a data word $\sigma$, we write $\sigma_{i}$ for the $i$ th element (couple) of the word, and $\pi_{1}, \pi_{2}$ for the projections on $\Sigma$ and $D$. A valuation is defined as a partial 
map $v:\{1, \ldots, n\} \rightarrow D$. The satisfaction relation $\models$ is inductively defined (we omitted $\mathrm{X}^{-1}$ and $\mathrm{F}^{-1}$ for succinctness):

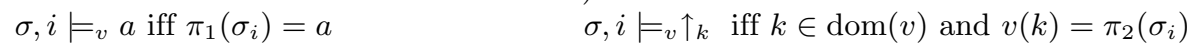

$\sigma, i \models_{v} \downarrow_{k} \varphi$ iff $\sigma, i \mid=_{v\left[k \mapsto \pi_{2}\left(\sigma_{i}\right)\right]} \varphi \quad \sigma, i \models_{v} \mathrm{X} \varphi$ iff $i<|\sigma|$ and $\sigma, i+1=_{v} \varphi$

$\sigma, i \models_{v} \mathrm{~F} \varphi$ iff for some $i \leq j \leq|\sigma|$ we have $\sigma, j \models_{v} \varphi$

where $1 \leq i \leq|\sigma|$. We denote $\sigma, i \models \models_{\emptyset} \varphi$ by $\sigma, i \models \varphi$, with $v_{\emptyset}$ the empty valuation; and we write $\sigma=\varphi$ for $\sigma, 1=\varphi$. Also, in the case of $\mathrm{LTL}_{1}^{\downarrow}$ we use $\downarrow$ and $\uparrow$ instead of $\downarrow_{1}$ and $\uparrow_{1}$ for simplicity.

In Section 5 we will lift our results to trees and XPath. In order to do this it is convenient to introduce now a restriction of $L T L_{1}^{\downarrow}$ such that with this restriction $\mathrm{LTL}_{1}^{\downarrow}$ corresponds to XPath. A formula of $\mathrm{LTL}_{1}^{\downarrow}$ is said to be simple if (i) there is at most one occurrence of $\uparrow$ within the scope of each occurrence of $\downarrow$ and, (ii) there is no negation between an occurrence of $\uparrow$ and its matching $\downarrow$, except maybe immediately before $\uparrow$. We denote by $s L T L_{1}^{\downarrow}$ the fragment of $L_{T L} L_{1}^{\downarrow}$ containing only simple formulas. The correspondence between $\mathrm{sLTL}_{1}^{\downarrow}$ and XPath will be made explicit in Proposition 1 of Section 5.

Faulty counter systems. For proving our lower bounds we will use a reduction from faulty counter automata that we describe here. A counter automaton (CA) with zero testing is a tuple $\left\langle\Sigma, Q, q_{0}, n, \delta, F\right\rangle$, where $\Sigma$ is a finite alphabet, $Q$ is a finite set of states, $q_{0}$ is the initial state, $n \in \mathbb{N}$ is the number of counters, $\delta \subset Q \times \Sigma \times L \times Q$ is the transition relation over the instruction set $L=$ \{inc, dec, ifzero $\} \times\{1, \ldots, n\}$, and $F \subset Q$ is the set of accepting states. A counter valuation is a function $v:\{1, \ldots, n\} \rightarrow \mathbb{N}$. An error-free run over $w \in$ $\Sigma^{*}$ is a finite sequence $\left\langle q_{0}, v_{0}\right\rangle \stackrel{w_{0}, \ell_{0}}{\longrightarrow}\left\langle q_{1}, v_{1}\right\rangle \stackrel{w_{1}, \ell_{1}}{\longrightarrow} \ldots$ observing the standard interpretation of the instructions $\ell_{0}, \ell_{1}, \cdots(\langle\operatorname{dec}, c\rangle$ can only be performed if $c$ is nonzero), where $v_{0}, v_{1}, \ldots$ are counter valuations, $v_{0}$ assigns 0 to each counter, and $w=w_{0} w_{1} \ldots$ such that $w_{i} \in \Sigma \cup\{\varepsilon\}$ for every $i$. A run is accepting iff it ends with an accepting state.

A Minsky CA has error-free runs. For these automata, already with only two counters, finitary emptiness is undecidable 14. An Incrementing CA (from now on ICA) is defined as a Minsky CA except that its runs may contain errors that increase one or more counters non-deterministically. We write that two valuations are in the relation $v \leq v^{\prime}$ iff, for every counter $c, v(c) \leq v^{\prime}(c)$. Runs of Incrementing CA are defined by replacing the relation $\stackrel{a, \ell}{\rightarrow}$ by $\stackrel{a, \ell}{\rightsquigarrow}$, where $\langle p, u\rangle \stackrel{a, \ell}{\rightsquigarrow}$ $\langle q, v\rangle$ iff there exist valuations $u^{\prime}, v^{\prime}$ such that $u \leq u^{\prime}, v^{\prime} \leq v$ and $\left\langle p, u^{\prime}\right\rangle \stackrel{a, \ell}{\rightarrow}\left\langle q, v^{\prime}\right\rangle$.

In [8, Theorem 2.9] it is shown that the results of [16] and [15] on Channel Machines can be adapted to prove the following result.

Theorem 1. Emptiness of ICA is decidable and non primitive recursive.

\section{The case of $s L T L \perp$}

In this section we show that satisfiability of $\operatorname{sLTL}_{1}^{\downarrow}\left(\mathrm{F}_{\mathrm{s}}\right)$ is non primitive recursive over data words. We then prove that satisfiability is undecidable for 
$\operatorname{sLTL}_{1}^{\downarrow}\left(\mathrm{F}_{\mathrm{s}}, \mathrm{F}_{\mathrm{s}}{ }^{-1}\right)$. In the next section we will show that the logic $L T L_{1}^{\downarrow}(\mathrm{F})$ is also non primitive recursive and that $\operatorname{LTL}_{1}^{\downarrow}\left(F, F^{-1}\right)$ is undecidable. In Section 5 we will use the results of this section for proving lower bounds for several fragments of XPath over data trees.

\subsection{Lower bound for $\operatorname{sLTL}_{1}^{\downarrow}\left(\mathrm{F}_{\mathrm{s}}\right)$.}

Theorem 2. Satisfiability of $\mathrm{s} L T L_{1}^{\downarrow}\left(\mathrm{F}_{\mathrm{s}}\right)$ on data words is non primitive recursive.

Proof. We exhibit a PTIme reduction from the non-emptiness of ICA to satisfiability of $\mathrm{sLTL}_{1}^{\downarrow}\left(\mathrm{F}_{\mathrm{s}}\right)$. Let $C=\left\langle\Sigma, Q, q_{0}, n, \delta, F\right\rangle$ be an ICA.

Let $L=\left\{\left(\text { inc }_{i}\right)_{1 \leq i \leq n},\left(\operatorname{dec}_{i}\right)_{1 \leq i \leq n},\left(\text { ifzero }_{i}\right)_{1 \leq i \leq n}\right\}$, and $\hat{\Sigma}=Q \times(\Sigma \cup\{\varepsilon\}) \times$ $L \times Q$. We construct a formula $\varphi_{C} \in \mathrm{sLTL}_{1}^{\downarrow}\left(\mathrm{F}_{\mathrm{s}}\right)$ that is satisfied by a data word iff $C$ accepts the word. We view a run of $C$ of the form:

as a string in $\hat{\Sigma}$ :

$$
\left\langle q_{0}, v_{0}\right\rangle \stackrel{a, \text {,inc }_{i}}{\rightsquigarrow}\left\langle q_{1}, v_{1}\right\rangle \stackrel{b, \text { dec }_{j}}{\rightsquigarrow}\left\langle q_{2}, v_{2}\right\rangle \stackrel{b, \text { ifzero }_{i}}{\rightsquigarrow}\left\langle q_{3}, v_{3}\right\rangle \cdots
$$

$$
\left\langle q_{0}, a, \text { inc }_{i}, q_{1}\right\rangle\left\langle q_{1}, b, \operatorname{dec}_{j}, q_{2}\right\rangle\left\langle q_{2}, b, \text { ifzero }_{i}, q_{3}\right\rangle \cdots
$$

The formula $\varphi_{C}$ will force that any string that satisfies it codes a run of $C$. In order to do this, $\varphi_{C}$ must ensure that:

(begin) the string starts with $q_{0}$,

(end) the string ends with a state of $F$,

(tran) every symbol of $\hat{\Sigma}$ in the string corresponds to a transition of $C$,

(chain) the last component of a symbol of $\hat{\Sigma}$ is equal to the first component of the next symbol,

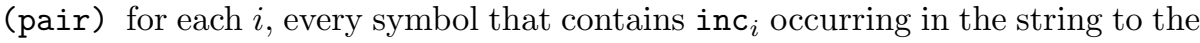
left of a symbol containing ifzero ${ }_{i}$, can be paired with a symbol containing $\operatorname{dec}_{i}$ and occurring in between the inc $_{i}$ and the ifzero ${ }_{i}$.

Before continuing let us comment on the (pair) condition. If we were coding runs of a perfect Minsky CA (ie, with no incremental errors), to the left of any position containing a ifzero ${ }_{i}$, we would require a perfect matching between inc $i$ and $\operatorname{dec}_{i}$ operations in order to make sure that the value of the counter $i$ is indeed zero at the position of the test. But as we are dealing with ICA, we only need to check that each inc $i$ has an associated $\operatorname{dec}_{i}$ to its right and before the test for zero, but we do not enforce the converse, that all $\operatorname{dec}_{i}$ match an inc $_{i}$. This is fortunate because this would require past navigational operators.

The first difficulty comes from the fact that (pair) is not a regular relation. The pairing will be obtained using data values. The second difficulty is to enforce (chain) without having access to the string successor relation. In order to simulate the successor relation we add extra symbols to the alphabet, with suitable associated data values.

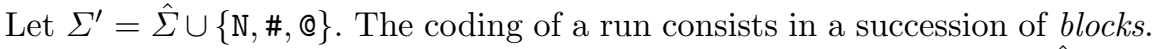
Each block is a sequence of 3 or 4 symbols, "c N\#" or "c @ N\#", with $c \in \hat{\Sigma}$. The data value associated to the $c$ and \# symbols of a block is the same and uniquely 
determines the block: no two blocks may have the same data value. The data value associated with the symbol $\mathrm{N}$ of a block is the data value of the next block. If a block contains a symbol $c$ that codes a inc in $_{i}$ operation that is later in the string followed by a ifzero $i$, then this block contains a symbol @ whose data value is the that of the block containing $\operatorname{dec}_{i}$ that is paired with $c$.

For instance in the example of Fig. 1 one can see four blocks $b_{1}, b_{2}, b_{3}, b_{4}$. Each of them starts with a symbol from $\hat{\Sigma}$ coding a transition of the ICA and ends with a \# with the same data value marking the end of the block. Inside the block, the data value of $\mathrm{N}$ is the same as the data value of the next block. The data value of @ corresponds to that of a future block. In this example $c_{1}$ must correspond to a inc $c_{i}$ while $c_{3}$ to $\operatorname{dec}_{i}$ and there must be a ifzero ${ }_{i}$ somewhere to the remaining part of the word (say, $b_{5}$ ). Moreover $c_{2}$ can't be a ifzero $i$ as otherwise the data value of the symbol @ would refer to a block to the left of $c_{2}$.

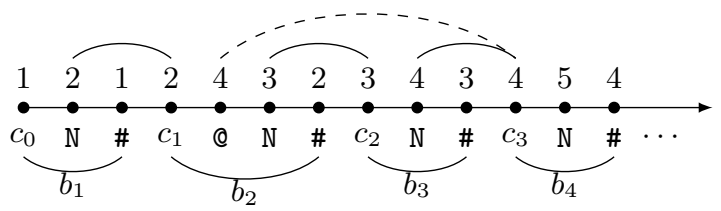

Fig. 1. Coding of an ICA run.

We now show that the coding depicted above can be enforced in $\operatorname{sLTL}_{1}^{\downarrow}\left(\mathrm{F}_{\mathrm{s}}\right)$. By $\pi_{1}, \pi_{2}, \pi_{3}, \pi_{4}$ we denote the projection of each symbol of $\hat{\Sigma}$ into its corresponding component. To simplify the presentation we use the following abbreviations:

$$
\begin{aligned}
& \hat{\sigma} \equiv \bigvee_{c \in \hat{\Sigma}} c \\
& \operatorname{INC}(i) \equiv \bigvee_{c \in \hat{\Sigma}, \pi_{3}(c)=\operatorname{inc}_{i}} c \\
& \mathrm{INC} \equiv \bigvee_{i} \mathrm{INC}(i) \\
& \operatorname{DEC}(i) \equiv \bigvee_{c \in \hat{\Sigma}, \pi_{3}(c)=\operatorname{dec}_{i}} c \\
& \text { LAST } \equiv \hat{\sigma} \wedge \neg \mathrm{F}_{\mathrm{s}} \hat{\sigma} \\
& \mathrm{IZ}(i) \equiv \bigvee_{c \in \hat{\Sigma}, \pi_{3}(c)=\text { ifzero }_{i}} c
\end{aligned}
$$

The formula $\varphi_{C}$ that we build is the conjunction of all the folowing formulas.

Forcing the structure

$\mathrm{G}\left(\mathrm{LAST} \Rightarrow \neg \mathrm{F}_{\mathrm{s}} \mathrm{T}\right)$ : The string ends with the last transition,

$\bigwedge_{c \in\{\mathrm{N}, \mathbb{e}, \#\}} \mathrm{G}\left(c \Rightarrow \neg\left(\downarrow \mathrm{F}_{\mathrm{s}}(c \wedge \uparrow)\right)\right)$ : the data value associated to each $\mathrm{N}$, \# and @ uniquely determines the occurrence of that symbol,

$\mathrm{G}\left((\hat{\sigma} \wedge \neg \mathrm{LAST}) \Rightarrow\left(\downarrow \mathrm{F}_{\mathrm{s}}\left(\mathrm{N} \wedge \mathrm{F}_{\mathrm{s}}(\# \wedge \uparrow)\right)\right)\right)$ : each occurrence of $\hat{\Sigma}$ (except the last one) is in a block that contains a $N$ and then a \#,

$\bigwedge_{i} \mathrm{G}\left(\left(\mathrm{INC}(i) \wedge \mathrm{F}_{\mathrm{s}}(\mathrm{IZ}(i))\right) \Rightarrow \downarrow \mathrm{F}_{\mathrm{s}}\left(\mathbb{C} \wedge \mathrm{F}_{\mathrm{s}}\left(\mathrm{N} \wedge \mathrm{F}_{\mathrm{s}}(\# \wedge \uparrow)\right)\right)\right)$ : every inc $i$ block to the left of a ifzero ${ }_{i}$ must have a @ before the $\mathrm{N}$,

$\mathrm{G}\left((\hat{\sigma} \wedge \neg \mathrm{INC}) \Rightarrow \neg \downarrow \mathrm{F}_{\mathrm{s}}\left(\mathbb{Q} \wedge \mathrm{F}_{\mathrm{s}}(\# \wedge \uparrow)\right)\right):$ only blocks inc are allowed to have a $\mathbb{Q}$,

$\bigwedge_{s \in\{\mathrm{N}, \Theta\}} \mathrm{G}\left(\hat{\sigma} \Rightarrow \neg\left(\downarrow \mathrm{F}_{\mathrm{s}}\left(s \wedge \mathrm{F}_{\mathrm{s}}\left(s \wedge \mathrm{F}_{\mathrm{s}}(\# \wedge \uparrow)\right)\right)\right)\right)$ : there is at most one occurrence of $\mathrm{N}$ and $@$ in each block,

$\bigwedge_{s \in \hat{\Sigma} \cup\{\#\}} \mathrm{G}\left(\hat{\sigma} \Rightarrow \neg \downarrow \mathrm{F}_{\mathrm{s}}\left(s \wedge \mathrm{F}_{\mathrm{s}}(\# \wedge \uparrow)\right)\right.$ : there is exactly one symbol \# and one symbol of $\hat{\Sigma}$ per block,

$\mathrm{G}\left(\mathrm{N} \Rightarrow \downarrow \mathrm{F}_{\mathrm{s}}\left(\# \wedge \mathrm{F}_{\mathrm{s}}(\hat{\sigma} \wedge \uparrow)\right)\right)$ : each symbol N's datum points to a block to its right, 
$\mathrm{G}\left(\mathrm{N} \Rightarrow \neg \downarrow \mathrm{F}_{\mathrm{s}}\left(\# \wedge \mathrm{F}_{\mathrm{s}}\left(\# \wedge \mathrm{F}_{\mathrm{s}}(\hat{\sigma} \wedge \uparrow)\right)\right)\right)$ : in fact $\mathrm{N}$ has has to point be the next block.

Once the structure has the expected shape, we can enforce the run as follows. All the formulas below are based on the following trick. In a test of the form $\downarrow \mathrm{F}_{\mathrm{s}}\left(\mathrm{N} \wedge \mathrm{F}_{\mathrm{s}}(\# \wedge \uparrow)\right)$ which is typically performed at a position of symbol $\hat{\Sigma}$, the last symbol \# must have the same data value as the initial position. Hence, because of the structure above, both must be in the same block. Thus the middle symbol $\mathrm{N}$ must also be inside that block. From the structure we know that the data value of this $\mathrm{N}$ points to the next block. Therefore by replacing the test $\mathrm{N}$ by one of the form $N \wedge\left(\downarrow \mathrm{F}_{\mathbf{s}}(\uparrow \wedge s)\right)$ we can transfer some finite information from the current block to the next one. This gives the desired successor relation.

Forcing a run

(begin)

(end)

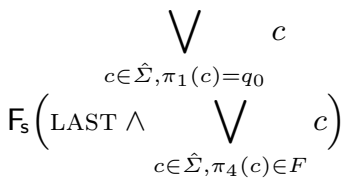

(tran) All elements used from $\hat{\Sigma}$ correspond to valid transitions. Let $\hat{\Sigma}^{C}$ be that set of transitions of $C$,

$$
\mathrm{G}\left(\bigwedge_{c \in \hat{\Sigma} \backslash \hat{\Sigma}^{C}} \neg c\right)
$$

(chain) For every $c \in \hat{\Sigma}$,

$$
\mathrm{G}\left(c \Rightarrow\left(\downarrow \mathrm{F}_{\mathrm{s}}\left(\mathrm{N} \wedge \mathrm{F}_{\mathrm{s}}(\# \wedge \uparrow) \wedge \bigvee_{\substack{d \in \hat{\Sigma}, \pi_{4}(c)=\pi_{1}(d)}}\left(\downarrow \mathrm{F}_{\mathrm{s}}(d \wedge \uparrow)\right)\right)\right)\right)
$$

(pair) We first make sure that the block of the @ of an inc $c_{k}$ is matched with a block of a $\operatorname{dec}_{k}$ :

$$
\bigwedge_{k} \mathrm{G}\left(\operatorname{INC}(k) \Rightarrow\left(\neg \downarrow \mathrm{F}_{\mathrm{s}}\left(@ \wedge \mathrm{F}_{\mathrm{s}}(\# \wedge \uparrow)\right) \vee \downarrow \mathrm{F}_{\mathrm{s}}\left(@ \wedge \downarrow \mathrm{F}_{\mathrm{s}}(\operatorname{DEC}(k) \wedge \uparrow) \wedge \mathrm{F}_{\mathrm{s}}(\# \wedge \uparrow)\right)\right)\right)
$$

Now, every inc $_{k}$ block to the left of a ifzero ${ }_{k}$ block:

- 1. The block must contain an @ element:

$$
\bigwedge_{k} \mathrm{G}\left(\mathrm{INC}(k) \Rightarrow\left(\downarrow \mathrm{F}_{\mathrm{s}}\left(@ \wedge \mathrm{F}_{\mathrm{s}}(\# \wedge \uparrow)\right) \vee \neg \mathrm{F}_{\mathrm{s}}(\mathrm{IZ}(k))\right)\right)
$$

- 2. The data value of that @ element must point to a future block before any occurrence of ifzero :

$$
\bigwedge_{k} \mathrm{G}\left(\operatorname{INC}(k) \Rightarrow \neg\left(\downarrow \mathrm{F}_{\mathrm{s}}\left(@ \wedge \downarrow \mathrm{F}_{\mathrm{s}}\left(\mathrm{IZ}(k) \wedge \mathrm{F}_{\mathrm{s}} \uparrow\right) \wedge \mathrm{F}_{\mathrm{s}}(\# \wedge \uparrow)\right)\right)\right)
$$

This concludes the construction of $\varphi_{C}$. The correctness proof is standard.

\subsection{Undecidability of $\operatorname{sLTL}_{1}^{\downarrow}\left(\mathrm{F}_{\mathrm{s}}, \mathrm{F}_{\mathrm{s}}{ }^{-1}\right)$}

We now consider $\operatorname{sLTL}_{1}^{\downarrow}\left(\mathrm{F}_{\mathrm{s}}, \mathrm{F}_{\mathrm{s}}{ }^{-1}\right)$. The extra modality can be used to code the run of a (non faulty) Minsky CA.

Theorem 3. Satisfiability of $\mathrm{sLTL}_{1}^{\downarrow}\left(\mathrm{F}_{\mathrm{s}}, \mathrm{F}_{\mathrm{s}}{ }^{-1}\right)$ is undecidable. 
Proof. Consider a Minsky CA $C$. We revisit the proof of Theorem 2 It is easy to see that we can enforce the absence of faulty increments during the run by asking that every $\operatorname{dec}_{i}$ element is referenced by some previous inc $c_{i}$ block: $\bigwedge_{i} \mathrm{G}\left(\operatorname{DEC}(i) \Rightarrow \downarrow \mathrm{F}_{\mathrm{s}}{ }^{-}(@ \wedge \uparrow)\right)$. We thus make sure that every dec is related to a corresponding inc. Hence, the coding is that of a perfect (non faulty) run.

\section{The case of $\mathrm{LTL}_{1}^{\downarrow}$}

In this section we lift the lower bounds of the previous section by considering the temporal operator $F$ instead of $F_{s}$. We can only do so by removing at the same time the restriction to simple formulas. Hence the results of this section cannot be applied to XPath. Notice that $L T L_{1}^{\downarrow}(F)$ and $\operatorname{sLTL}_{1}^{\downarrow}\left(\mathrm{F}_{\mathrm{s}}\right)$ are incomparable in terms of expressive power. Indeed, properties like $\downarrow F(a \wedge \uparrow \wedge F(b \wedge \uparrow))$ cannot be expressed in $\operatorname{sLTL}_{1}^{\downarrow}\left(\mathrm{F}_{\mathrm{s}}\right)$, while $L T L_{1}^{\downarrow}(\mathrm{F})$ cannot express that the model has at least two elements. We do not know whether $\operatorname{sLTL}_{1}^{\downarrow}(\mathrm{F})$, which is weaker than the two above mentioned logics, is already non primitive recursive. The results of this section improve the results of $[8]$ which show that satisfiability is non primitive recursive for $L_{T L}^{\downarrow}(X, F)$ and undecidable for $L_{1} L_{1}^{\downarrow}\left(X, F, F^{-1}\right)$.

Theorem 4. Over data words,

1. Satisfiability of $\mathrm{LTL}_{1}^{\downarrow}(\mathrm{F})$ is decidable and non primitive recursive.

2. Satisfiability of $\mathrm{LTL}_{1}^{\downarrow}\left(\mathrm{F}, \mathrm{F}^{-1}\right)$ is undecidable.

Proof. We only prove Item 1 , the proof of Item 2 being similar.

Consider an ICA $C$ and recall the coding of runs of $C$ used in the proof of Theorem 2. In the construction of the formula $\varphi_{C}$, whenever we have " $s \wedge \mathrm{F}_{\mathrm{s}}\left(s^{\prime} \wedge\right.$ $\varphi)$ " for some $\varphi$ and $s \neq s^{\prime}$ two different symbols, $F_{s}$ can be equivalently replaced by the $\mathrm{F}$ temporal operator. This is the case in all formulas except in three places: (i) The formula saying that $\mathrm{N}$ should point to the next block contains $\# \wedge F_{s}(\# \wedge \varphi)$. But from the structure that is enforced, this can equivalently be replaced by \# $\wedge \mathrm{F}(\mathrm{N} \wedge \mathrm{F}(\# \wedge \varphi))$. (ii) To enforce that each block contains at most one occurrence of symbols in $\hat{\Sigma} \cup\{\mathrm{N}, \#, \mathbb{Q}\}$. (iii) To enforce that no two symbols in $\hat{\Sigma} \cup\{\mathrm{N}, \#, @\}$ have the same data value.

In order to cope with (ii) and (iii), we use a slightly different coding for runs of $C$. This coding is the same as the one for the proof of Theorem 2 except that we allow succession of equal symbols, denoted as group in the sequel. Note that in a group two different occurrences of the same symbol in general may have different data values, as we can no longer enforce their distinctness. However, as we will see, we can enforce that the $\hat{\Sigma}$ group of elements of a block have all the same data value.

Hence a block is now either a group of $c \in \hat{\Sigma}$ followed by a group of $\mathrm{N}$ followed by a group of \# or the same with a group of $@$ in between. A coding of a run is depicted in Figure 2 .

This structure is enforced by modifying the formulas of the proof of Theorem 2 as follows. 


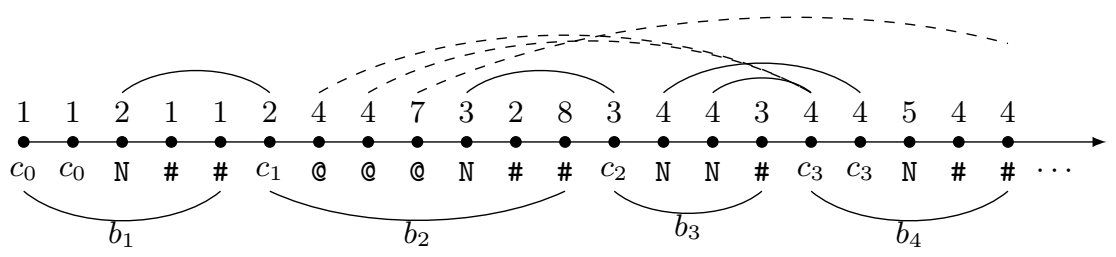

Fig. 2. $\mathrm{LTL}_{1}^{\downarrow}(\mathrm{F})$ cannot avoid having repeated consecutive symbols.

(i) The formulas that limit the number of occurrences of symbols in a block are replaced by formulas limiting the number of groups in a block.

(ii) The formulas requiring that no two occurrences of a same symbol may have the same data values are replaced by formulas requiring that no two occurrences of a same symbol in different groups have the same data value.

(iii) In all other formulas, $F_{s}$ is replaced by $F$.

(iv) Finally, we ensure that although we may have repeated symbols inside a block, all symbols from $\hat{\Sigma}$ have the same data value.

$$
\mathrm{G}(\hat{\sigma} \Rightarrow \neg \downarrow \mathrm{F}((\hat{\sigma} \vee \#) \wedge \neg \uparrow \wedge \mathrm{F}(\# \wedge \uparrow)))
$$

Note that this implies that each $\mathrm{N}$ of a group must have the same data values as they all point to the next block. However there could still be $\odot$ symbols with different data values as depicted in Fig. 2.

The new sentences now imply, for instance, that:

1. Every position from a group of $c \in \hat{\Sigma}$ have the same data value which is later matched by an element of a group of \#.

2. Every position from a group of $\mathrm{N}$ has the same data value as a position $c \in \hat{\Sigma}$ of the next block (and then, it has only one possible data value).

3. Every position from a group @ has the same data value as a position $c \in \hat{\Sigma}$ of a block to its right. Note that the data values of two $@$ of the same group may correspond to the data values of symbols in different blocks. This is basically the main conceptual difference with the previous proof.

The proof of correctness of the construction is left to the reader.

Note that in the previous proof we used the fact that $L T L_{1}^{\downarrow}(F)$, although it has only one register, can make (in)equality tests several times throughout a path (as used in the formula of item (iv) in the proof), something that $s L T L_{1}^{\downarrow}\left(F_{s}\right)$ and XPath cannot do.

Two registers. When 2 registers are available the previous result can be adapted to code a (non faulty) Minsky CA with a strategy similar to [주 , Theorem 5.4].

Theorem 5. Satisfiability of $\mathrm{LTL}_{2}^{\downarrow}(\mathrm{F})$ is undecidable over data words. 


\section{Data trees and XPath}

We now turn to data trees. An XML document can be seen as an unranked tree with attributes and data values in its nodes. While a data tree has only one data value per node, an XML document element may have several attributes each of which with an associated data value. All XPath fragments treated in this paper can force all elements of an XML document to have only one attribute. Therefore all hardness results in the present work hold also for the class of XML documents.

The logic $\mathrm{XPath}$. XPath is a two-sorted language, with path expressions $(\alpha, \beta, \ldots)$ and node expressions $(\varphi, \psi, \ldots)$. These are defined by mutual recursion:

$$
\begin{gathered}
\alpha, \beta::=\downarrow\left|\downarrow^{+}\right| \uparrow\left|\uparrow^{+}\right| \rightarrow\left|\rightarrow^{+}\right| \leftarrow\left|+_{\leftarrow}\right| \varepsilon|\alpha \beta| \alpha \cup \beta \mid \alpha[\varphi] \\
\varphi, \psi::=\sigma|\langle\alpha\rangle| \neg \varphi|\varphi \wedge \psi| \alpha=\beta \mid \alpha \neq \beta \quad(\sigma \in \Sigma)
\end{gathered}
$$

A path expression essentially describes a traversal of the tree by using the axis: child $(\downarrow)$, descendant $\left(\downarrow^{+}\right)$, the next-sibling $(\rightarrow)$, following-sibling $\left(\rightarrow^{+}\right)$ and their inverses, with the ability to test at any stage for node conditions. Let $t$ be a data tree with domain $T$ and labeling function $\lambda$. The semantics of XPath is defined by induction is the usual intuitive way, we only give here some cases:

$$
\begin{array}{ll}
\llbracket \downarrow \rrbracket^{t}=\{(x, x i) \mid x i \in T\} & \llbracket \alpha \beta \rrbracket^{t}=\left\{(x, z) \mid \exists y \cdot(x, y) \in \llbracket \alpha \rrbracket^{t} \wedge(y, z) \in \llbracket \beta \rrbracket^{t}\right\} \\
\llbracket\langle\alpha\rangle \rrbracket^{t}=\left\{x \in T \mid \exists y \cdot(x, y) \in \llbracket \alpha \rrbracket^{t}\right\} & \llbracket \alpha[\varphi] \rrbracket^{t}=\left\{(x, y) \in \llbracket \alpha \rrbracket^{t} \mid y \in \llbracket \varphi \rrbracket^{t}\right\} \\
\llbracket \varepsilon \rrbracket^{t}=\{(x, x) \mid x \in T\} & \llbracket \alpha^{+} \rrbracket^{t}=\text { the transitive closure of } \llbracket \alpha \rrbracket^{t} \\
\llbracket \alpha=\beta \rrbracket^{t}=\left\{x \in T \mid \exists y, z .(x, y) \in \llbracket \alpha \rrbracket^{t},(x, z) \in \llbracket \beta \rrbracket^{t}, \pi_{2}(\lambda(y))=\pi_{2}(\lambda(z))\right\} \\
\llbracket \alpha \neq \beta \rrbracket^{t}=\left\{x \in T \mid \exists y, z .(x, y) \in \llbracket \alpha \rrbracket^{t},(x, z) \in \llbracket \beta \rrbracket^{t}, \pi_{2}(\lambda(y)) \neq \pi_{2}(\lambda(z))\right\}
\end{array}
$$

A key property for using results of Section 3 is that simple formulas can be translated to XPath and back. The proof of this result is straightforward by induction on the formula.

Proposition 1. Over data words, $\operatorname{sLTL} \downarrow 1\left(\mathrm{~F}_{\mathrm{s}}\right)$ and $\mathrm{XPath}\left(\rightarrow^{+},=\right)$have the same expressive power. The same holds for $\mathrm{sLTL}_{1}^{\downarrow}\left(\mathrm{F}_{\mathrm{s}}, \mathrm{F}_{\mathrm{s}}{ }^{-1}\right)$ and $\mathrm{XPath}\left({ }_{\leftarrow}^{+}, \rightarrow^{+},=\right)$. Moreover, in both cases, the transformation from $\mathrm{sLTL}_{1}^{\downarrow}$ to $\mathrm{XPath}$ takes polynomial time while it takes exponential time in the other direction.

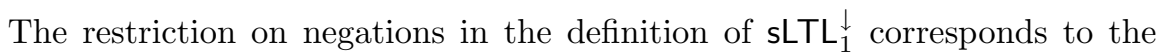
fact that XPath path expressions are always positive: any path $\alpha$ is essentially a nesting of operators $\mathrm{F}$ with intermediate tests. We remark that there is a big difference between $\mathrm{XPath}\left(\rightarrow^{+},=\right)$over data words and $\operatorname{XPath}\left(\downarrow^{+},=\right)$over data trees. Indeed $\mathrm{XPath}\left(\downarrow^{+},=\right)$is closed under bisimulation and hence it cannot assume that the tree is a vertical path. As the string structure was essential in the proof of Theorem 2 the non primitive recursiveness of $\mathrm{XPath}\left(\rightarrow^{+},=\right)$over data words does not lift to $\mathrm{XPath}\left(\downarrow^{+},=\right)$over data trees. Actually, satisfiability of $\operatorname{XPath}\left(\downarrow^{+},=\right)$is ExpTime-complete 10. However, if one considers the $\operatorname{logic} \operatorname{XPath}\left(\downarrow^{+}, \rightarrow,=\right)$ then the axis $\rightarrow$ can be used to enforce a vertical path $\left(\neg \downarrow^{+}[\rightarrow]\right)$ and therefore it follows from Theorem 2 and Proposition 1 that:

Corollary 1. Satisfiability of $\mathrm{XPath}\left(\downarrow^{+}, \rightarrow,=\right)$ on data trees is at least non primitive recursive. 
Similarly, in XPath $\left(\downarrow^{+}, \uparrow^{+},=\right)$one can simulate a string by going down to a leaf using $\downarrow^{+}$and then use the path from that leaf to the root as a string using $\uparrow^{+}$.

Corollary 2. Satisfiability of $\mathrm{XPath}\left(\downarrow^{+}, \uparrow^{+},=\right)$on data trees is at least non primitive recursive.

Note that the decidability of $\operatorname{XPath}\left(\downarrow^{+}, \rightarrow,=\right)$ and of $\operatorname{Xath}\left(\downarrow^{+}, \uparrow^{+},=\right)$is still an open problem.

Open problem: Is XPath $\left(\downarrow^{+}, \uparrow^{+},=\right)$decidable over data trees?

It would be interesting to know whether the strictness of the axis $\downarrow^{+}$is necessary in the above two results. This boils down to know whether $\operatorname{sLTL}_{1}^{\downarrow}(\mathrm{F})$ is already not primitive recursive over data words. Note that the proof of Theorem 4 uses in an essential way the possibility to make (in)equality tests several times throughout a path. This is exactly what cannot be expressed in $\operatorname{sLTL}_{1}^{\downarrow}(F)$.

Open problem: Is satisfiability of $s L^{\prime} L_{1}^{\downarrow}(F)$ primitive recursive over data words?

We conclude with some simple consequences of Theorem 3 and Proposition 1 ;

Corollary 3. Satisfiability of XPath $\left(+_{\leftarrow}^{+}, \rightarrow^{+},=\right)$and of XPath $\left(\downarrow^{+}, \uparrow^{+}, \rightarrow,=\right)$ over data trees is undecidable.

Corollary 4. Satisfiability of $\mathrm{XPath}\left(\rightarrow^{+}, \downarrow, \uparrow,=\right)$ is undecidable.

Proof. This is similar to the proof of Theorem 3 with a slight difference. Consider that the coding of the run of the counter machine is done at the first level of the tree (i.e., at distance 1 from the root). Then, the property to ensure that every decrement has a corresponding increment is now: $\bigwedge_{i} \neg \downarrow[\operatorname{DEC}(i) \wedge \neg \varepsilon=\uparrow \downarrow[@]]$.

\section{Discussion}

By 8] it is known that satisfiability of $\operatorname{LTL}_{1}^{\downarrow}(\mathrm{X}, \mathrm{F})$ with infinite data words is undecidable. The proof of Theorem 4 can be extended to code runs of ICA over infinite data words, which is known to be undecidable, to show that this result already holds in the absence of $\mathrm{X}$.

Theorem 6. On infinite data words, the satisfiability problem of $\mathrm{LTL}_{1}^{\downarrow}(\mathrm{F})$ is undecidable.

\section{Summary of results}

In the table below we summarize the main results and some of the consequences we have mentioned. In this table, $\overline{\mathrm{PR}}$ stands for non primitive recursive. 


\begin{tabular}{|c|c|c|}
\hline Logic & Complexity & Details \\
\hline $\mathrm{LTL}_{1}^{\downarrow}(\mathrm{F})$ & $\overline{\mathrm{PR}}$, decidable & Theorem $4 \& 8$ \\
\hline $\mathrm{LTL}_{1}^{\downarrow}\left(\mathrm{F}, \mathrm{F}^{-1}\right)$ & undecidable & Theorem 4 \\
\hline $\mathrm{LTL}_{2}^{\downarrow}(\mathrm{F})$ & undecidable & Theorem 5 \\
\hline $\mathrm{sLTL}_{1}^{\downarrow}\left(\mathrm{F}_{\mathrm{s}}\right)$ & $\overline{\mathrm{PR}}$, decidable & $\begin{array}{ll}\text { Theorem } 2 \text { \& } \\
\end{array}$ \\
\hline $\mathrm{sLTL}_{1}^{\downarrow}\left(\mathrm{F}_{\mathrm{s}}, \mathrm{F}_{\mathrm{s}}^{-1}\right)$ & undecidable & Theorem 3 \\
\hline $\mathrm{XPath}\left(\downarrow^{+}, \rightarrow,=\right)$ & $\overline{\mathrm{PR}}$, decidability unknown & Corollary 1 \\
\hline $\operatorname{XPath}\left(\downarrow^{+}, \uparrow^{+},=\right)$ & $\overline{\mathrm{PR}}$, decidability unknown & Corollary 2 \\
\hline $\operatorname{XPath}\left(\downarrow^{+}, \uparrow^{+}, \rightarrow,=\right)$ & undecidable & Corollary 3 \\
\hline $\operatorname{XPath}\left(\rightarrow^{+}, \downarrow, \uparrow,=\right)$ & undecidable & Corollary 4 \\
\hline
\end{tabular}

\section{References}

1. Michael Benedikt, Wenfei Fan, and Floris Geerts. XPath satisfiability in the presence of DTDs. J. ACM, 55(2), 2008.

2. Henrik Björklund, Wim Martens, and Thomas Schwentick. Optimizing conjunctive queries over trees using schema information. In MFCS, pages 132-143, 2008.

3. Mikolaj Bojańczyk, Claire David, Anca Muscholl, Thomas Schwentick, and Luc Segoufin. Two-variable logic on data trees and XML reasoning. In PODS, pages 10-19, 2006.

4. Mikolaj Bojańczyk, Anca Muscholl, Thomas Schwentick, Luc Segoufin, and Claire David. Two-variable logic on words with data. In LICS, pages 7-16, 2006.

5. Patricia Bouyer, Antoine Petit, and Denis Thérien. An algebraic approach to data languages and timed languages. Inf. Comput., 182(2):137-162, 2003.

6. J. Clark and S. DeRose. XML path language (XPath). Website, 1999. W3C Recommendation. http://www.w3.org/TR/xpath

7. Claire David. Complexity of data tree patterns over XML documents. In MFCS, pages 278-289, 2008.

8. Stéphane Demri and Ranko Lazić. LTL with the freeze quantifier and register automata. ACM Transactions on Computational Logic, 2009.

9. Stéphane Demri, Ranko Lazić, and David Nowak. On the freeze quantifier in constraint LTL: Decidability and complexity. In TIME, pages 113-121, 2005.

10. Diego Figueira. Satisfiability of downward XPath with data equality tests. In PODS, Providence, Rhode Island, USA, 2009. ACM Press.

11. Floris Geerts and Wenfei Fan. Satisfiability of XPath queries with sibling axes. In $D B P L$, volume 3774, pages 122-137. Springer, 2005.

12. Marcin Jurdziński and Ranko Lazić. Alternating automata on data trees and XPath satisfiability. CoRR, abs/0805.0330, 2008.

13. Alexei Lisitsa and Igor Potapov. Temporal logic with predicate lambdaabstraction. In TIME, pages 147-155, 2005.

14. Marvin L. Minsky. Computation: finite and infinite machines. Prentice-Hall, Inc., 1967.

15. Joel Ouaknine and James Worrell. On Metric temporal logic and faulty Turing machines. In FoSSACS, volume 3921, pages 217-230, 2006.

16. Philippe Schnoebelen. Verifying lossy channel systems has nonprimitive recursive complexity. Information Processing Letters, 83(5):251-261, September 2002. 


\section{A Missing proofs}

\section{A.1 Correctness of the construction of the proof of Theorem 2}

Given an ICA $C$, the proof exhibited a formula $\varphi_{C} \in \operatorname{XPath}\left(\rightarrow^{*},=\right)$ that is satisfied by a data word iff $C$ accepts the word. We show here that the construction is correct.

From data word to an accepting ICA run Let $w$ be a data word satisfying $\varphi_{C}$. Let $B_{1} B_{2} \cdots B_{m}$ be the decomposition of $w$ into blocks as described above. Let $c_{i}=\left\langle q_{i}, a_{i}, \ell_{i}, q_{i+1}\right\rangle$ be the element of $B_{i}$ in $\hat{\Sigma}$. We show that $w^{\prime}=a_{1} a_{2} \cdots a_{m}$ is accepted by $C$. We construct a run of $C$ on $w^{\prime}$ such that $C$ is in state $q_{i}$ before reading letter $a_{i}$ of $w^{\prime}$ and execute the transitions $c_{i}$. By (tran) each one of these are valid transitions of the ICA, and (chain) ensures that the sequence is consistent. (begin) takes care of the initialization condition and (end) of the accepting condition. It remains to construct the valuation $v_{1}, \ldots, v_{m+1}$ of the counter at each step such that we have $\left\langle q_{i}, v_{i}\right\rangle \stackrel{a_{i}, \ell_{i}}{\varkappa}\left\langle q_{i+1}, v_{i+1}\right\rangle$ as desired.

As expected $v_{1}$ is the zero valuation. We construct the rest of the valuations by induction, simulating a perfect counter machine and introducing incrementing errors in a lazy way, whenever necessary. At step $i$, given a transition $\left\langle q_{i}, a_{i}, \ell_{i}, q_{i+1}\right\rangle$ and the current valuation $v_{i}$ we set $v_{i+1}$ such that $\left\langle q_{i}, v_{i}\right\rangle \stackrel{a_{i}, \ell_{i}}{\leadsto}$ $\left\langle q_{i+1}, v_{i+1}\right\rangle$.

- If $\ell_{i}$ is inc in $_{k}$, then $v_{i+1}:=v_{i}\left[k \mapsto v_{i}(k)+1\right]$ and clearly $\left\langle q_{i}, v_{i}\right\rangle \stackrel{a_{i}, \ell_{i}}{\longrightarrow}\left\langle q_{i+1}, v_{i+1}\right\rangle$.

- If $\ell_{i}$ is $\operatorname{dec}_{k}$ then:

- If $v_{i}(k)>0$ then $v_{i+1}:=v_{i}\left[k \mapsto v_{i}(k)-1\right]$.

- If $v_{i}(k)=0$, then $v_{i+1}:=v_{i}$.

In both cases one can verify that $\left\langle q_{i}, v_{i}\right\rangle \stackrel{a_{i}, \ell_{i}}{\leadsto}\left\langle q_{i+1}, v_{i+1}\right\rangle$, the transition being with an incrementing error of 1 in the second case.

- If $\ell_{i}$ is ifzero $_{k}$, we set $v_{i+1}:=v_{i}$. To ensure that $\left\langle q_{i}, v_{i}\right\rangle \stackrel{a_{i}, \ell_{i}}{\sim}\left\langle q_{i+1}, v_{i+1}\right\rangle$ we must show that necessarily $v_{i}(k)=0$. This is a consequence of condition pair. This condition enforces that each inc $c_{k}$ must be paired with a unique $\operatorname{dec}_{k}$ to its right and before the ifzero ${ }_{k}$ test. Hence the lazy strategy above ensures that each increment is decremented later on and before the zero test.

From an accepting ICA run to data word Given an accepting run of the $\mathrm{ICA}\left\langle q_{1}, v_{1}\right\rangle \stackrel{a_{1}, \ell_{1}}{\leadsto}\left\langle q_{2}, v_{2}\right\rangle \stackrel{a_{2}, \ell_{2}}{\leadsto} \ldots \stackrel{a_{m}, \ell_{m}}{\leadsto}\left\langle q_{m+1}, v_{m+1}\right\rangle$ we construct a data word $w$ verifying $\varphi_{C}$ as follows. Let $c_{i}$ be $\left\langle q_{i}, a_{i}, \ell_{i}, q_{i+1}\right\rangle$. $w$ consists of a concatenation of $m$ blocks $B_{1} B_{2} \cdots B_{m}$ each one defined:

- If $i=m, B_{i}$ is the word $c_{i}$ with data value $m$.

- If $i<m$, and $\ell_{i} \in\left\{\operatorname{dec}_{k}\right.$, ifzero $\left.{ }_{k}\right\}$, then set $B_{i}$ to $c_{i} \mathrm{N \#}$ with respective data values $i, i+1, i$.

$$
\left\langle c_{i}, i\right\rangle\langle\mathrm{N}, i+1\rangle\langle \#, i\rangle
$$


- If $i<m$ and $\ell_{i}$ is inc in $_{k}$. Consider the minimal $d>i$ such that $v_{d+1}(k)=v_{i}(k)$ and set $B_{i}$ to

- $c_{i} @ \mathrm{~N} \#$ with respective data values $i, d, i+1, i$ if $d$ exists

- $c_{i} \mathrm{~N}$ \# with respective data values $i, i+1, i$ if not.

This construction assigns the unique data value $i$ to each block $B_{i}$, and set the data value of each symbol $\mathrm{N}$ to the data value of the next block $B_{i+1}$. The constraints (begin), (end), (tran), (chain) are obviously true. It remains to verify the constraints on @: they must have a unique data value, must point to a corresponding dec instruction that occur before any corresponding zero test, and no two @ may point to the same dec instruction. Consider a position $i$ such that $\ell_{i}$ is inc $_{k}$ with a subsequent zero test ifzero ${ }_{k}$ at position $j>i$. Because the zero test is correct, the increment of counter $k$ made at step $i$ must be decremented at some position between $i$ and $j$. Hence there is a $i<d<j$ such that $v_{d+1}(k)=v_{i}(k)$. By construction the data value of the @ symbol of $B_{i}$ is the minimal such $d$. By minimality, $c_{d}$ must be a $\operatorname{dec}_{k}$ instruction. Assume

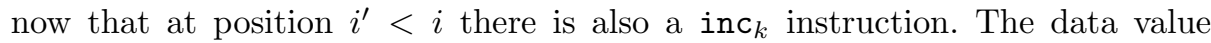
of the @ symbol of $B_{i^{\prime}}$ cannot be $d$. Because if this would be the case then $v_{i^{\prime}}(k)=v_{d+1}(k)=v_{i}(k)$ and $d$ would not be minimal for the position $i^{\prime}$. Hence (pair) is also satisfied and $w \models \varphi_{C}$.

\section{A.2 Proof of Theorem 4,2}

Recall the statement.

Theorem 4,2 Over data words, Satisfiability of $\operatorname{LTL}_{1}^{\downarrow}\left(\mathrm{F}, \mathrm{F}^{-1}\right)$ is undecidable.

Proof (sketch). Consider a Minsky Counter Automaton $C$. We revisit the previous proof. We modify the coding of a run of $C$ by also inserting a group of symbols @ in each dec block. Using $\mathrm{F}^{-1}$, we add formulas ensuring that every block containing a dec instruction also contains a group of @ symbols such that their data values refer to previous blocks containing a matching inc instruction. We also make sure that two @ from different blocks have different data values. All this can be done by taking the $\mathrm{F}^{-1}$ counterpart of the formulas we constructed with F.

We show that the new formula $\varphi_{C}$ is satisfied by a data word iff $C$ accept the word. Constructing a data word that satisfies $\varphi_{C}$ from a word accepted by $C$ is done as in the proof of Theorem 2

For the other direction one needs to show that from a word that satisfies $\varphi_{C}$, the corresponding word with the obvious run and obvious valuations of the counter is accepting for $C$. One can verify that the new extra conditions do imply that no null counter is ever decreased and each zero test is correct, based on the fact that for every $\operatorname{dec}_{i}$ there must be at least one inc $c_{i}$ to its left, and for every inc ${ }_{i}$ there must have a related $\operatorname{dec}_{i}$ before any ifzero ${ }_{i}$ test. 


\section{A.3 Proof of Theorem 5}

First recall the statement of Theorem 5

Theorem 5 Satisfiability of $\mathrm{LTL}_{2}^{\downarrow}(\mathrm{F})$ is undecidable over data words.

Proof. We adapt the proof of part 1 . of Theorem 4 using ideas already present in 813] for coding Minsky CA with only forward temporal operators. Fix a two counter machine $C=\left\langle\Sigma, Q, q_{0}, 2, \delta, F\right\rangle$. The main idea is to modify the coding of Theorem 4 by adding in each block a symbol $\mathrm{h}_{1}$ whose data value is supposed to be the one of the last inc in $_{1}$ that has previously occurred, a symbol $l_{1}$ whose data value is supposed to be the one of the last inc l $_{1}$ that has not been decreased yet and similarly with $\mathrm{h}_{2}$ and $\mathrm{l}_{2}$ for the second counter. A zero test of counter 1 can then only occur in a block where the data values of $h_{1}$ and $l_{1}$ match. The coding is depicted in Figure 3 .

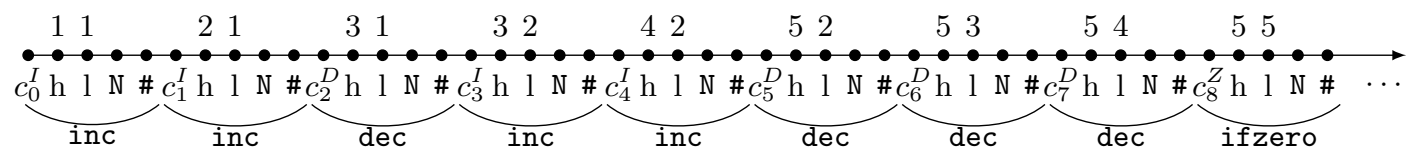

Fig. 3. Coding of a 1-counter machine run. Only data values of the $\mathrm{h}$ and $\mathrm{l}$ symbols are represented for the sake of clarity. Only one symbol per group is also represented. Elements depicted as $c_{i}^{I}, c_{i}^{D}$ and $c_{i}^{Z}$ correspond, respectively, to increment, decrement and zero testing symbols of $\hat{\Sigma}$.

Based on these ideas, the formula $\varphi_{C}$ that we construct makes sure that:

(i) The structure. We enforce a structure that is a sequence of blocks of the form " $\left\langle q, w, l, q^{\prime}\right\rangle \mathrm{h}_{1} \mathrm{l}_{1} \mathrm{~h}_{2} \mathrm{l}_{2} \mathrm{N \# ",} \mathrm{with} \mathrm{the} \mathrm{possibility} \mathrm{that} \mathrm{there} \mathrm{are} \mathrm{repeated}$ consecutive symbols. This can be done in the same way we did before.

As for the previous codings, we demand that in each block, for every element in the first group of $\hat{\Sigma}$ symbols there is an element in the last group of symbols \# with the same data value. This is enforce with a formula of $\operatorname{LTL}_{1}^{\perp}(\mathrm{F})$ as before.

With the help of the second register we can now enforce that each group of symbols has the same data value, for all symbol $s$ we have the formula:

$$
\mathrm{G}\left(\hat{\sigma} \Rightarrow \downarrow_{1} \neg \mathrm{F}\left(s \wedge \downarrow_{2} \mathrm{~F}\left(s \wedge \neg \uparrow_{2} \wedge \mathrm{F}\left(\# \wedge \uparrow_{1}\right)\right)\right)\right)
$$

(ii) Each $\left\langle q, w, l, q^{\prime}\right\rangle$ occurring in the string is in $\delta$. For the first one $q=q_{0}$, and for the last one $q^{\prime} \in F$. For any $\left\langle q, w, l, q^{\prime}\right\rangle$ and $\left\langle q^{\prime \prime}, w^{\prime}, l^{\prime}, q^{\prime \prime \prime}\right\rangle$ in consecutive blocks, $q^{\prime}=q^{\prime \prime}$. This can be checked just as in the previous codings.

(iii) In the initial block, the data values of $h_{1}$ and $l_{1}$ are the same and the data values of $h_{2}$ and $l_{2}$ are the same (we only show the formula for $h_{1}$ and $l_{1}$ ):

$$
\downarrow_{1} \neg \mathrm{F}\left(\mathrm{h}_{1} \wedge \downarrow_{2} \mathrm{~F}\left(\mathrm{l}_{1} \wedge \neg \uparrow_{2} \wedge \mathrm{F}\left(\# \wedge \uparrow_{1}\right)\right)\right)
$$


(iv) In any block immediately after a ifzero instruction, the data values of $\mathrm{h}_{1}, \mathrm{l}_{1}, \mathrm{~h}_{2}, \mathrm{l}_{2}$ are identical to those of the ifzero instruction. We only give the formula for ifzero ${ }_{1}$ instruction with the $\mathrm{h}_{1}$ symbol.

$\neg \mathrm{F}\left(\mathrm{IZ}(1) \wedge \downarrow_{1} \mathrm{~F}\left(\mathrm{~h}_{1} \wedge \downarrow_{2} \mathrm{~F}\left(\psi \wedge \mathrm{F}\left(\# \wedge \uparrow_{1}\right)\right)\right)\right)$

where $\psi \equiv \mathrm{N} \wedge \downarrow_{1} \mathrm{~F}\left(\mathrm{~h}_{1} \wedge \neg \uparrow_{2} \wedge \mathrm{F}\left(\# \wedge \uparrow_{1}\right)\right)$

(v) In any block immediately after an inc $c_{1}$ instruction, $\mathrm{h}_{1}$ is not in the same class as any preceding $h_{1}$ (stated in the formula below) and $l_{1}, h_{2}, l_{2}$ are in the same class as in the previous block (this can be stated using a formula similar to the one for for item (iv)).

$\neg \mathrm{F}\left(\mathrm{h}_{1} \wedge \downarrow_{1} \mathrm{~F}\left(\mathrm{INC}(1) \wedge \downarrow_{2} \wedge \mathrm{F}\left(\varphi \wedge \mathrm{F}\left(\# \wedge \uparrow_{2}\right)\right)\right)\right)$

where $\varphi \equiv \mathrm{N} \wedge \downarrow_{2} \mathrm{~F}\left(\uparrow_{2} \wedge \mathrm{F}\left(\mathrm{h}_{1} \wedge \uparrow_{1} \wedge \mathrm{F}\left(\# \wedge \uparrow_{2}\right)\right)\right)$

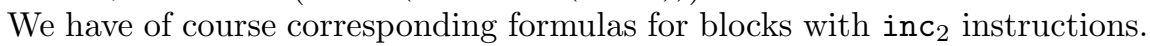

(vi) In any $\operatorname{dec}_{1}$ block, $\mathrm{h}_{1}$ and $\mathrm{l}_{1}$ have different data values.

$$
\neg \mathrm{F}\left(\mathrm{DEC}(1) \wedge \downarrow_{1} \mathrm{~F}\left(\mathrm{~h}_{1} \wedge \downarrow_{2} \mathrm{~F}\left(\mathrm{l}_{1} \wedge \uparrow_{2} \wedge \mathrm{F}\left(\# \wedge \uparrow_{1}\right)\right)\right)\right)
$$

We have a corresponding formula for blocks with a dec $c_{2}$ instruction.

(vii) In any ifzero ${ }_{1}$ block, $h_{1}$ and $l_{1}$ have the same data value. This can be expressed with a formula as above. We have a corresponding formula for ifzero 2 blocks.

(viii) In any block immediately after a $\operatorname{dec}_{1}$ block $B$,

- $\mathrm{h}_{1}$ is in the same class as the previous $\mathrm{h}_{1}$, and

$-l_{1}$ is in the same class as the $h_{1}$ occurring in a block immediately after the rightmost block (occurring before $B$ ) with a $\mathrm{h}_{1}$ that is in the same class as the $l_{1}$ of $B$.

- $\mathrm{h}_{2}$ and $\mathrm{l}_{2}$ are in the same class as their corresponding symbol of $B$.

The first and third conditions can be expressed using formulas similar to those above. The difficult part is to enforce the second condition. For this we introduce some macros:

$$
\begin{aligned}
\operatorname{InBlock}_{i}(\varphi) \equiv & \downarrow_{i} \mathrm{~F}\left(\varphi \wedge \mathrm{F}\left(\# \wedge \uparrow_{i}\right)\right) \\
\operatorname{NextBlock}_{i}(\varphi) \equiv & \downarrow_{i} \mathrm{~F}\left(\mathrm{~N} \wedge \mathrm{F}\left(\# \wedge \uparrow_{i}\right) \wedge\right. \\
& \left.\downarrow_{i} \mathrm{~F}\left(\hat{\sigma} \wedge \varphi \wedge \mathrm{F}\left(\# \wedge \uparrow_{i}\right)\right)\right) \\
\operatorname{InNextBlock}_{i}(\varphi) \equiv & \operatorname{NextBlock}_{i}\left(\operatorname{InBlock}_{i}(\varphi)\right)
\end{aligned}
$$

The second condition can then be stated:

$$
\begin{aligned}
\neg \mathrm{F}\left(\hat { \sigma } \wedge \downarrow _ { 2 } \mathrm { F } \left(\mathrm { h } _ { 1 } \wedge \downarrow _ { 1 } \mathrm { F } \left(\mathrm{N} \wedge \mathrm{F}\left(\# \wedge \uparrow_{2}\right) \wedge\right.\right.\right. & \\
& \left.\left.\left.\downarrow_{2} \mathrm{~F}\left(\hat{\sigma} \wedge \uparrow_{2} \wedge \mathrm{F}\left(\mathrm{h}_{1} \wedge \neg \uparrow_{1} \wedge \downarrow_{2} \varphi\right)\right)\right)\right)\right)
\end{aligned}
$$

where $\varphi \equiv \mathrm{F}\left(\operatorname{DEC}(i) \wedge \operatorname{InBlock}_{2}\left(\mathrm{l}_{1} \wedge \uparrow_{1}\right) \wedge \operatorname{InNextBlock}_{1}\left(\mathrm{l}_{1} \wedge \neg \uparrow_{2}\right)\right)$

We have a corresponding formula for blocks following a $\operatorname{dec}_{2}$ instruction. 


\section{A.4 Proof of Proposition 1}

Proposition 1 Over data words, $\mathrm{sLTL}_{1}^{\downarrow}\left(\mathrm{F}_{\mathrm{s}}\right)$ and $\mathrm{XPath}\left(\rightarrow^{+},=\right)$have the same expressive power. The same hold for $\operatorname{sLTL}_{1}^{\downarrow}\left(\mathrm{F}_{\mathrm{s}}, \mathrm{F}_{\mathrm{s}}{ }^{-1}\right)$ and $\mathrm{XPath}\left({ }^{+} \leftarrow, \rightarrow^{+},=\right)$. More-

over, in both cases, the transformation from $\mathrm{sLTL}_{1}^{\downarrow}$ to XPath takes polynomial time while it takes exponential time in the other direction.

Proof (sketch). The translation from $\mathrm{sLTL}_{1}^{\downarrow}$ to XPath is straightforward by induction. One then naturally obtain equality tests of the form $\varepsilon=\alpha$ or $\varepsilon \neq \alpha$ depending on whether the unique possible negation is present or not.

The other translation is also by induction. The non trivial part concerns the translation of node expressions of the form $\alpha=\beta$. Over data words, one of the path expressions $\alpha$ or $\beta$ must end first, say $\alpha$. In this case the node expression can be decomposed into a disjunction of (exponentially many) expressions that first start with a path expression that merge $\alpha$ with the part $\beta_{1}$ of $\beta$ that is common to with $\alpha$, followed by a test of the form $\varepsilon=\beta_{2}$, where $\beta=\beta_{1} \beta_{2}$. Tests of the form $\varepsilon=\beta_{2}$ are the immediate to translate into $\mathrm{LTTL}_{1}^{\downarrow}$.

\section{B Complete semantics of XPath}

Recall that XPath is a two-sorted language, with path expressions $(\alpha, \beta, \ldots)$ and node expressions $(\varphi, \psi, \ldots)$. These are defined by mutual recursion:

$$
\begin{gathered}
\alpha::=\downarrow||^{+}|\uparrow| \uparrow^{+}|\rightarrow| \rightarrow^{+}|\leftarrow|{ }^{+} \leftarrow|\varepsilon| \alpha \beta|\alpha \cup \beta| \alpha[\varphi] \\
\varphi::=\sigma|\langle\alpha\rangle| \neg \varphi|\varphi \wedge \psi| \alpha=\beta \mid \alpha \neq \beta \quad(\sigma \in \Sigma)
\end{gathered}
$$

A path expression essentially describes a traversal of the tree by using the axis: child $(\downarrow)$, descendant $\left(\downarrow^{+}\right)$, the next-sibling $(\rightarrow)$, following-sibling $\left(\rightarrow^{+}\right)$ and their inverses, with the ability to test at any stage for node conditions. Let $t$ be a data tree with domain $T$ and labeling function $\lambda$. The semantics of XPath is defined by induction as follows:

$$
\begin{aligned}
\llbracket \downarrow \rrbracket^{t} & =\{(x, x i) \mid x i \in T\} \\
\llbracket \uparrow \rrbracket^{t} & =\{(x i, x) \mid x i \in T\} \\
\llbracket \leftarrow \rrbracket^{t} & =\{(x i, x(i-1)) \mid x(i-1) \in T\} \\
\llbracket \rightarrow \rrbracket^{t} & =\{(x i, x(i+1)) \mid x(i+1) \in T\} \\
\llbracket \alpha^{+} \rrbracket^{t} & =\text { the transitive closure of } \llbracket \alpha \rrbracket^{t} \\
\llbracket \varepsilon \rrbracket^{t} & =\{(x, x) \mid x \in T\} \\
\llbracket \alpha \beta \rrbracket^{t} & =\left\{(x, z) \mid \exists y \cdot(x, y) \in \llbracket \alpha \rrbracket^{t} \wedge(y, z) \in \llbracket \beta \rrbracket^{t}\right\} \\
\llbracket \alpha \cup \beta \rrbracket^{t} & =\llbracket \alpha \rrbracket^{t} \cup \llbracket \beta \rrbracket^{t} \\
\llbracket \alpha[\varphi] \rrbracket^{t} & =\left\{(x, y) \in \llbracket \alpha \rrbracket^{t} \mid y \in \llbracket \varphi \rrbracket^{t}\right\} \\
\llbracket \sigma \rrbracket^{t} & =\left\{x \in T \mid \pi_{1}(\lambda(x))=\sigma\right\} \\
\llbracket\langle\alpha\rangle \rrbracket^{t} & =\left\{x \in T \mid \exists y \cdot(x, y) \in \llbracket \alpha \rrbracket^{t}\right\}
\end{aligned}
$$




$$
\begin{aligned}
& \llbracket \neg \varphi \rrbracket^{t}= T \backslash \llbracket \varphi \rrbracket^{t} \\
& \llbracket \varphi \wedge \psi \rrbracket^{t}=\llbracket \varphi \rrbracket^{t} \cap \llbracket \psi \rrbracket^{t} \\
& \llbracket \alpha=\beta \rrbracket^{t}=\left\{x \in T \mid \exists y, z \cdot(x, y) \in \llbracket \alpha \rrbracket^{t},\right. \\
&\left.(x, z) \in \llbracket \beta \rrbracket^{t}, \pi_{2}(\lambda(y))=\pi_{2}(\lambda(z))\right\} \\
& \llbracket \alpha \neq \beta \rrbracket^{t}=\left\{x \in T \mid \exists y, z \cdot(x, y) \in \llbracket \alpha \rrbracket^{t},\right. \\
&\left.(x, z) \in \llbracket \beta \rrbracket^{t}, \pi_{2}(\lambda(y)) \neq \pi_{2}(\lambda(z))\right\}
\end{aligned}
$$

